\title{
FUNCTIONS OF EXPONENTIAL TYPE AND SEPARATED SEQUENCES
}

\author{
DAVID C. ULLRICH
}

(Communicated by J. Marshall Ash)

Let us say that the sequence of complex numbers $\left(\lambda_{j}\right)$ is separated if there exists $\varepsilon>0$ such that $\left|\lambda_{j}-\lambda_{k}\right| \geq \varepsilon \quad(j \neq k)$. For $0<p<\infty$ let $E^{p}$ denote the class of entire functions of exponential type whose restriction to the real line lies in the Lebesgue space $L^{p}$. A subharmonicity argument establishes the following classical result:

Theorem A. Suppose $\left(\lambda_{j}\right)$ is a separated sequence of complex numbers with bounded imaginary parts. Then

$$
\sum\left|f\left(\lambda_{j}\right)\right|^{p}<\infty
$$

for every $f \in E^{p}$.

(Theorem 17 on p. 96 of [Y] asserts this under the additional hypothesis that the $\lambda_{j}$ be real, but the argument in [Y] proves the slightly more general statement above.)

The purpose of this note is to point out that this result is sharp in a certain sense, settling a question posed on p. 221 of [Y]:

Theorem 1. Suppose $p>0$ and $\left(\lambda_{j}\right)$ is a sequence of complex numbers satisfying (1) for each $f \in E^{p}$. Then the $\lambda_{j}$ have bounded imaginary parts and can be partitioned into a finite union of separated sequences.

The proof will be a simple example of what is sometimes referred to as a "gliding hump" argument:

Proof. Suppose the $\lambda_{j}$ satisfy (1) for every $f \in E^{p}$. We leave it to the reader to show that $\operatorname{Im}\left(\lambda_{j}\right)$ is bounded; this is analogous to the argument below, but simpler. Supposing this, we show that the $\lambda_{j}$ can be partitioned into a finite union of separated sequences. We need only show that the number of $\lambda_{j}$ with real part lying in the interval $[\beta, \beta+1]$ is bounded. Supposing that this is false, we shall construct an $f \in E^{p}$ for which (1) fails: Choose $A \geq 1$ so that $\left|\operatorname{Im}\left(\lambda_{j}\right)\right| \leq A$ for all $j$. Now choose $g \in E^{p}$ with $|g(z)| \geq 1$ for all $z \in \mathbf{C}$

Received by the editors July $6,1988$.

1980 Mathematics Subject Classification (1985 Revision). Primary 42A70; Secondary 30D55. 
with $|z| \leq 2 A$. We claim that if constants $c_{k}>0$ and $\beta_{k} \in \mathbf{R}$ are chosen properly, then $f(z)=\sum_{k} c_{k} g\left(z+\beta_{k}\right)$ will give the required example.

Note first that if we take $c_{k}$ decreasing to zero fast enough then we will have $f \in E^{p}$ regardless of our choice of $\left(\beta_{k}\right)$. Fix such a sequence $\left(c_{k}\right)$. Let $I_{\beta}=\left\{j: \operatorname{Re}\left(\lambda_{j}\right) \in[\beta, \beta+1]\right\}$, so that our hypothesis on the $\lambda_{j}$ becomes the statement that the cardinality of $I_{\beta}$ is unbounded. Suppose we have chosen $\beta_{1}, \ldots, \beta_{N}$ in such a way that

$$
\sum_{j \in I_{\beta_{k}}}\left|f_{N}\left(\lambda_{j}\right)\right|^{p}>1 \quad(k=1, \ldots, N),
$$

where we have written $f_{N}(z)=\sum_{k=1}^{N} c_{k} g\left(z+\beta_{k}\right)$. Now, Theorem A shows that $g(z)$ approaches zero as $z$ tends to infinity within the strip $|\operatorname{Im}(z)| \leq A$. Thus, merely to take $\beta_{N+1}$ large enough will accomplish two things: It will ensure that $\left(2_{N}\right)$ remains true, at least for $1 \leq k \leq N$, when $f_{N}$ is replaced by $f_{N+1}$, and it will ensure that $\left|f_{N}\left(\lambda_{j}\right)\right| \leq \frac{1}{2}$ for $j \in I_{\beta_{N+1}}$. If in addition to taking $\beta_{N+1}$ large we choose $\beta_{N+1}$ so that the cardinality of $I_{\beta_{N+1}}$ is sufficiently large (depending on $c_{N+1}$ and $p$ ) then we have

$$
\left(2_{N+1}\right) \quad \sum_{j \in I_{\beta_{k}}}\left|f_{N+1}\left(\lambda_{j}\right)\right|^{p}>1 \quad(k=1, \ldots, N+1) .
$$

Now fix $k$ and let $N$ tend to infinity: $\sum_{j \in I_{\beta_{k}}}\left|f\left(\lambda_{j}\right)\right|^{p} \geq 1$, so that

$$
\sum_{j}\left|f\left(\lambda_{j}\right)\right|^{p}=\infty
$$

\section{REFERENCES}

[Y] R. M. Young, An introduction to nonharmonic Fourier series, Academic Press, New York, 1980.

Department of Mathematics, Oklahoma State University, Stillwater, Oklahoma 74078 